\title{
Euclid mission status after mission critical design
}

Laureijs, R., Racca, G., Mellier, Y., Musi, P., Brouard, L., et al.

R. Laureijs, G. D. Racca, Y. Mellier, P. Musi, L. Brouard, T. Böenke, L. Gaspar Venancio, E. Maiorano, A. Short, P. Strada, B. Altieri, G. Buenadicha, X. Dupac, P. Gomez Alvarez, J. Hoar, R. Kohley, R. Vavrek, A. Rudolph, M. Schmidt, J. Amiaux, H. Aussel, M. Berthé, M. Cropper, J.-C. Cuillandre, C. Dabin, J. Dinis, R. Nakajima, T. Maciaszek, R. Scaramella, A. da Silva, I. Tereno, O. R. Williams, A. Zacchei, R. Azzollini, F. Bernardeau, J. Brinchmann, C. Brockley-Blatt, F. Castander, A. Cimatti, C. Conselice, A. Ealet, P. Fosalba, W. Gillard, L. Guzzo, H. Hoekstra, P. Hudelot, K. Jahnke, T. Kitching, L. Miller, J. Mohr, W. Percival, V. Pettorino, J. Rhodes, A. Sanchez, M. Sauvage, S. Serrano, R. Teyssier, J. Weller, J. Zoubian, "Euclid mission status after mission critical design," Proc. SPIE 11443, Space Telescopes and Instrumentation 2020: Optical, Infrared, and Millimeter Wave, 114430F (13 December 2020); doi: 10.1117/12.2563145 


\section{Euclid Mission Status after Mission Critical Design}

R. Laureijs*1, G. D. Racca ${ }^{1}$, Y. Mellier ${ }^{8,15}$, P. Musi ${ }^{12}$, L. Brouard ${ }^{4}$, T. Bönke ${ }^{1}$, L. Gaspar Venancio ${ }^{1}$, E. Maiorano ${ }^{1}$, A. Short ${ }^{1}$, P. Strada ${ }^{1}$, B. Altieri ${ }^{2}$, J., G. Buenadicha ${ }^{2}$, X. Dupac ${ }^{2}$, P. Gomez Alvarez ${ }^{2}$,

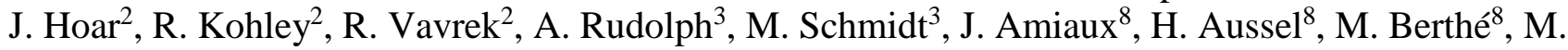
Cropper $^{11}$, J.-C. Cuillandre ${ }^{8}$, C. Dabin ${ }^{9}$, J. Dinis ${ }^{13}$, R. Nakajima ${ }^{5}$, T. Maciaszek ${ }^{9}$, R. Scaramella ${ }^{7}$, A. da Silva ${ }^{13}$, I. Tereno ${ }^{13}$, O.R. Williams ${ }^{14}$, A. Zacchei $^{6}$,

R. Azzolini ${ }^{11}$, F. Bernardeau ${ }^{15}$, J. Brinchman ${ }^{16}$, C. Brockley-Blatt ${ }^{11}$, F. Castander ${ }^{17}$, A. Cimatti ${ }^{20}$, C. Conselice $^{21}$, A. Ealet ${ }^{29}$, P. Fosalba ${ }^{17}$, W. Gillard ${ }^{18}$, L. Guzzo ${ }^{22}$, H. Hoekstra $^{23}$, P. Hudelot ${ }^{15}$, K. Jahnke $^{10}$, T. Kitching ${ }^{11}$, L. Miller ${ }^{24}$, J. Mohr $^{25}$, W. Percival ${ }^{26}$, V. Pettorino ${ }^{8}$, J. Rhodes ${ }^{19}$, A. Sanchez $^{27}$, M. Sauvage ${ }^{8}$, S. Serrano ${ }^{17}$, R. Teyssier ${ }^{28}$, J. Weller ${ }^{25}$, J. Zoubian ${ }^{18}$,

${ }^{1}$ European Space Agency / ESTEC, Keplerlaan 1, 2201 AZ Noordwijk, The Netherlands

${ }^{2}$ European Space Agency / ESAC, Villanueva de la Cañada, E-28692 Madrid, Spain

${ }^{3}$ European Space Agency / ESOC, Robert-Bosch-Str. 5, 64293 Darmstadt, Germany

${ }^{4}$ Airbus Defense \& Space, 31 rue des Cosmonautes, 31402 Toulouse, Cedex France

${ }^{5}$ Argelander-Institut für Astronomie, Auf dem Hügel 71, D-53121 Bonn

${ }^{6}$ INAF-Osservatorio di Trieste, Via G.B. Tiepolo 11, I-31131 Trieste, Italy

${ }^{7}$ INAF-Osservatorio di Roma, Via Frascati 33, I-00040 Monteporzio Catone (Roma), Italy

${ }^{8}$ IRFU, Service d'Astrophysique, CEA Saclay, F-91191 Gif-sur-Yvette Cedex, France

${ }^{9}$ Centre National d’Etudes Spatiales, 18 avenue Edouard Belin, F-31401 Toulouse Cedex 9, France

${ }^{10}$ Max Planck Institute for Astronomy, Koenigstuhl 17, D-69117, Heidelberg, Germany

${ }^{11}$ MSSL, University College London, Holmbury St Mary, Dorking, Surrey RH5 6NT, UK

${ }^{12}$ Thales Alenia Space Italia, Strada Antica di Collegno 253, 10146, Torino, Italy

${ }^{13}$ FCUL, University of Lisbon, Campo Grande, 1749-016 Lisboa, Portugal

${ }^{14}$ CIT, Univ. Groningen, Nettelbosje 1, 9747 AJ Groningen, The Netherlands

${ }^{15}$ CNRS-UPMC, Institut d’Astrophysique de Paris, 98B Bd Arago, F-75014 Paris

${ }^{16}$ Centro de Astrofisica da Universidade do Porto, Rua de Estrelas, 4150-762 Porto, Portugal

${ }^{17}$ IEEC, UAB, Gran Capita 2-4, 08034 Barcelona, Spain

${ }^{18}$ CPPM, Aix-Marseille Univ.Ave. de Luminy, 13009 Marseille, France

${ }^{19}$ JPL/Caltech, 4800 Oak Grove Dr, Pasadena, CA91109 USA

${ }^{20}$ Dipartimento di Fisica e Astronomia, Univ. di Bologna, Via Piero Gobetti 93/2, Bologna

${ }^{21}$ School of Physics and Astronomy, University of Nottingham, University Park, NG9 2RD, UK

${ }^{22}$ Dipartimento di Fisica,Università degli Studi di Milano, Via Celoria 16, 20133 Milano, Italy

${ }^{23}$ Sterrewacht, Univ. Leiden, Niels Bohrweg 2, 2333 CA Leiden, The Netherlands

${ }^{24}$ Dept. of Physics, Univ. Oxford, Keble Road, Oxford, OX1 3RH, UK

${ }^{25}$ Universitätssternwarte München, Scheinerstrasse 1, 81679 Munich, Germany

${ }^{26}$ WCA, Univ. of Waterloo, 200 Univ. Ave. West, Waterloo, Ontario, Canada N2L 3G1

${ }^{27}$ Max Planck Institute for Extraterrestrial Physics, Giessenbachstrasse 1, 85748 Garching Germany

${ }^{28}$ ICS, Univ. of Zurich, Winterthurerstrasse 190, 8057 Zurich, Switzerland

${ }^{29}$ IP2I, Bâtiment Paul Dirac, 4, Rue Enrico Fermi, 69622 Villeurbanne Cedex 


\begin{abstract}
Euclid, an ESA mission designed to characterise dark energy and dark matter, passed its Mission Critical Design Review in November 2018. It was demonstrated that the project is ready to start integration and test of the main systems, and that it has the ability to fulfil its top-level mission requirements. In addition, based on the performances at M-CDR, the scientific community has verified that the science requirements can be achieved for the Weak Lensing and Galaxy Clustering dark energy probes, namely a dark energy Figure of Merit of 400 and a 2\% accuracy in the growth factor exponent gamma. We present the status of the main elements of the Euclid mission in the light of the demanding high optical performance which is the essential design driver is the to meet the scientific requirements. We include the space segment comprising of a service module and payload module hosting the telescope and its two scientific instruments, and the ground segment, which encompasses the operational and science ground segment. The elements for the scientific success of the mission for a timely release of the data are shortly presented: the processing and calibration of the data, and the design of the sky survey. Euclid is presently on schedule for a launch in September 2022.
\end{abstract}

Keywords: Euclid, dark energy, dark matter, space telescope, cosmology, galaxies survey, data processing

\title{
1. INTRODUCTION
}

Euclid is a survey mission optimised for the measurement of two dark energy probes: Weak Lensing (WL) and Galaxy Clustering (GC). The experiment is to probe the Universe up to a redshift of $z=2$, equivalent to 10 billion years of cosmic history. The WL probe is a combination of deep optical imaging and near-infrared photometry in several bands. On a large WL sample of more than one billion galaxies the imaging is used to measure ellipticity and orientation of each galaxy and the photometry is used to determine its redshift. The GC probe applies slitless spectroscopy to measure the spectroscopic redshifts of more than 20 million galaxies from the $\mathrm{H}$-alpha emission line. At the high redshifts of $z>0.7$ the relevant signal of the galaxies is shifted into the near infrared $(\lambda>900 \mathrm{~nm})$. Euclid is required to survey the high galactic and high ecliptic regions on the sky with a total area of at least $15,000 \mathrm{deg}^{2}$.

Euclid has four scientific objectives: (1) Improve the dark energy figure of merit (DE-FOM) to a value in excess of 400, (2) test General Relativity on cosmic scales by determining the factor gamma for the growth of cosmic structures to a precision better than 2\%, (3) test the Cold Dark Matter paradigm and measure the neutrino mass with high precision, and (4) test the initial conditions of the concordance cosmological model. These objectives are achieved by combining the WL and GC probes. The combined DE-FOM is more than the sum of the figure of merits obtained by the individual probes, because they are governed by different biases. This makes Euclid a unique mission.

The main scientific driver for the design of the mission is the demanding optical performance on any position of the field of view of the instruments, to be achieved over a large survey area within the programmatic constraints. The optical performance must ensure a well-behaving and predictable point spread function (PSF) because a major fraction of the WL targets have intrinsic sizes that are only a few times larger than the size of the PSF. The driving requirements are the limits on the parameters characterising the PSF: full width at half maximum (FWHM), ellipticity (e) and size $\left(R^{2}\right)$ in the visual band, and the encircled energy radius $\left(r_{\mathrm{EE}}\right)$ in the infrared. The high level of knowledge needed to predict the shape of the PSF at the position of the galaxy has led to design adjustments and the need of an extensive in orbit calibration programme.

For a detailed and more quantitative design description of the mission at the time of the Mission Critical Design Review (M-CDR) milestone, which was passed in November 2018, we refer to [1]. The present paper concentrates on the subsequent developments which are in the interest of, or may have an impact on, the scientific requirements. We will give an outlook of the activities before and after launch in 2022.

This paper is organised as follows: we give an overview of the mission and program in section 2. In section 3 we describe the main space segment subsystems: the service module and payload module accommodating the telescope and instruments. In section 4, we present the status of the development of the mission operations, followed by the status of the science ground segment in section 5. In section 6 and 7 the preparations are presented for the calibration and survey operations, respectively. The outlook and conclusions are given in section 8. 


\section{MISSION OVERVIEW AND PROGRAMME}

Euclid is ESAs second Medium-Class mission of the Cosmic Vision programme, selected in 2011. It will be launched as a baseline with a Soyuz 2.1b/ST equipped with an upper stage Fregat from Centre Spatial Guyanais, ESA's spaceport near Kourou, French Guyana. The $1.2 \mathrm{~m}$ aperture space telescope is placed in a wide Lissajous orbit around the second Sun-Earth Lagrange point (SEL2) by direct orbit injection. The spacecraft can be launched throughout the year during a short daily time window around local noon. In its final orbit, the angle between the Earth-Spacecraft-Sun is determined by the exact time of launch. The launch time determines the closest angle of the Sun with respect to the uncovered telescope aperture, this angle is constrained to avoid damage of the payload due to excessive illumination. After a short cruise phase and a commissioning period of three months, Euclid will perform its 6 year nominal survey to achieve the science objectives.

The Euclid Consortium (EC) is a scientific organisation of more than 100 scientific institutes to provide to ESA the science requirements which includes the survey plan, the two scientific instruments VIS and NISP, and their part of the science ground segment (SGS).

As part of their partnership contribution, NASA provided the near-infrared detector systems, which include the sensor chip assemblies, cryo flex cables and the cold readout electronics, and SGS support, which includes a Science Data Centre.

To achieve the photometric accuracy for the WL probe, complementary photometry from ground based surveys is required. The provision of ground based data is outside the scope of ESA but is essential to reach the mission objectives. It is the responsibility of the EC to collect and procure the ground based photometry to obtain the photometric redshifts of the WL galaxies to the required accuracy.

\section{SPACE SEGMENT}

\subsection{Spacecraft and system}

The development of the space segment is headed by the Prime Contractor Thales Alenia Space (TAS) in Turin (Italy) leading a consortium of European companies. The spacecraft consists of a service module (SVM) and a payload module (PLM). The flight model of the PLM, developed and assembled by Airbus, will be delivered to TAS in Turin in mid 2021. The impact of the system optical performance on the spacecraft is given in Fig 1, which shows the elements that contribute to the errors in the PSF parameters.

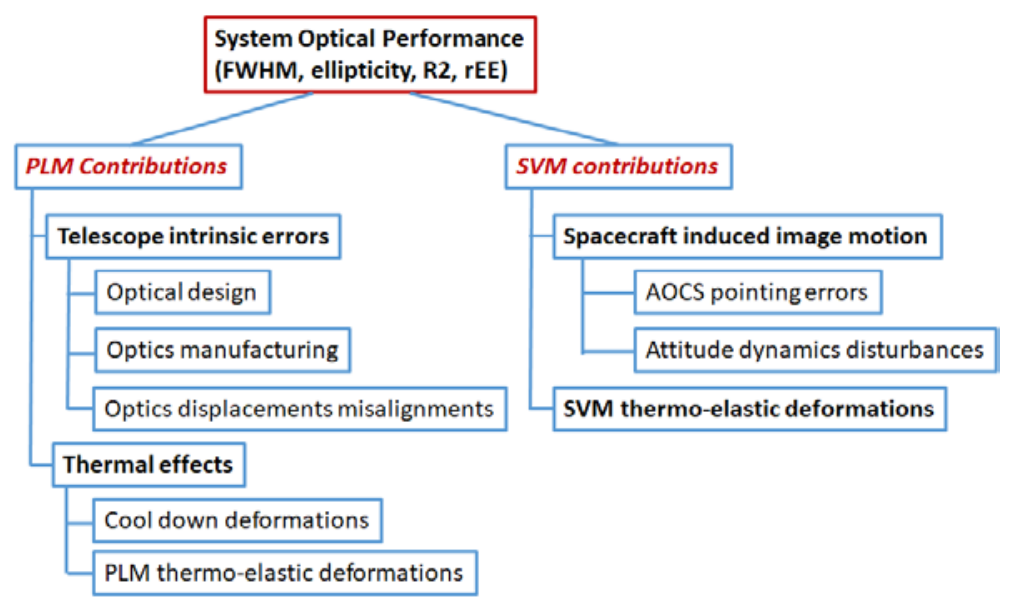

Figure 1. The impact of the PSF requirements on the system optical performance. The performance is affected by the different elements that contribute to the errors in the parameters that describe the point spread function. 
To analyse the static and dynamic PSF errors, a system thermo-optical performance (STOP) model was developed. The STOP analysis uses the thermal models of the SVM and the PLM with their interfaces to compute the temperatures in the telescope structure for more than 100 selected typical and extreme attitudes of the spacecraft in orbit with respect to the Sun, as well as a number of dynamic cases after a change in attitude. Aberrations of the telescope optics induced by these temperature changes were used to predict the optical performance of the telescope, by deriving the essential image parameters: ellipticity, R2 and FWHM of the PSF at typical positions in the focal plane. Among the inputs to the model were: several hundred CodeV telescope realisations, AOCS line of sight jitter timelines on a number of pointings, as well as micro-vibration time histories due to the filter wheels, shutter, high gain antenna, and reaction wheels actuations.

The structural thermal model (STM) of the Euclid spacecraft was tested in on the premises of Thales Alenia Space in Cannes, France, in 2019. The model consists of the qualification model of the SVM with sunshield and the structural thermal model of the PLM. The latter contained the SiC telescope structure, baseplate, the qualification model of the VIS instrument with thermal radiator and dummy loads representing the NISP instrument and its thermal radiator, see Fig 1. The STM has undergone the shock, vibration, and acoustic tests, which qualified the mechanical design of the spacecraft.

The results of the thermal tests were used to update the thermal mathematical model. In this way the model was able to provide results that showed a good correlation with the test results and could be used to predict the system thermooptical performance in flight with a good confidence.

During the assembly integration and test phases, and on the launch pad, special care shall be taken to keep the amount of particular and molecular contamination within requirements, to minimize straylight and loss of throughput. Study on the proper treatment of water contamination on ground and in orbit are ongoing, also considering the lessons learnt from Gaia. The success of these measures can only be validated in flight.

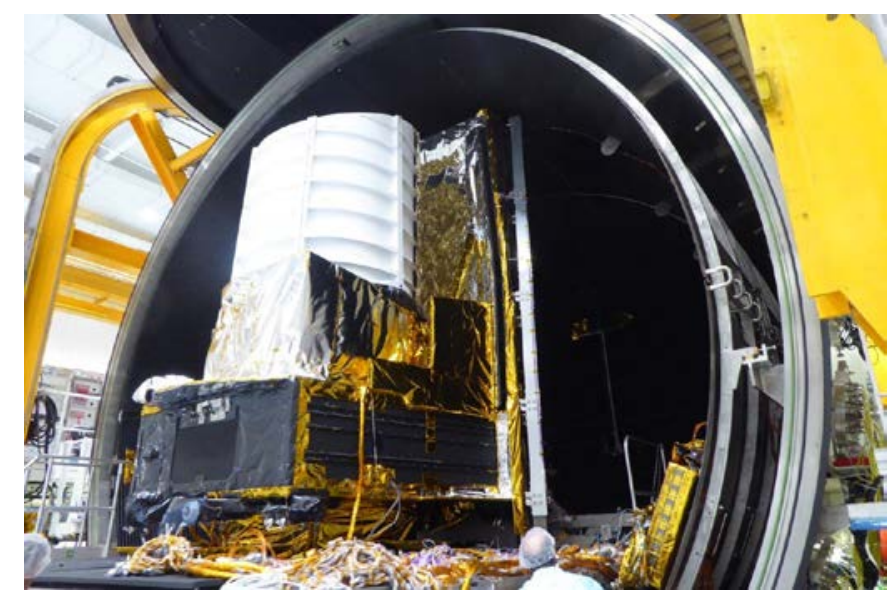

Figure 2. The Euclid structural thermal model (STM) rolling into the thermal vacuum test chamber at TAS-F in Cannes (France).

\subsection{Service module (SVM)}

The attitude and orbit control system (AOCS) consists of three star trackers mounted on the SVM and a fine guidance system with CCD sensors in the focal plane of the Euclid telescope. The pointing stability is achieved with cold gas which is contained in 4 pressurised nitrogen tanks, an optical gyro and reaction wheels. An additional reaction wheel is provided to compensate for the induced torque by the rotation of the grism and filter wheel movement in the infrared instrument.

All the flight model subsystems for the service module have been delivered to TAS-I in the course of 2019 and they are fully integrated on the spacecraft panels. The functional tests are ongoing.

The scientific data are transferred to ground using, for the first time in ESA mission, the CCSDS file delivery protocol (CFDP). The flight software and hardware implementation of CFDP alongside with its compatibility with ground elements has been verified on the Avionics Model. 


\subsection{Payload module (PLM)}

The PLM is developed and built by an industrial consortium under the leadership of Airbus Defence and Space (ADS) in Toulouse, France. The PLM features a full silicon carbide ( $\mathrm{SiC}$ ) optical baseplate, telescope truss and optical elements to facilitate the homothetic three mirror anastigmatic Korsch telescope [2]. The second mirror (M2) is mounted on a mechanism with three degrees of freedom to adjust the focus after launch. A dichroic mirror splits the light of the telescope by reflection to the VIS instrument, and by transmission to the NISP instrument. Dielectric coatings on the optical elements are applied to achieve the transmission in the optical band from 550-900 $\mathrm{nm}$ because the VIS instrument itself does not contain a separate bandpass filter, which would degrade the image quality. The PLM also ensures the temperature requirements and thermal stability of the telescope and instruments, by means of radiators and the telescope baffle which provides the thermal insulation of the telescope truss and mirrors. For a detailed description of the PLM performances we refer to [3].

Around the time of the Mission CDR, it was found that for the dichroic mirror the combination of the large diameter and wide optical (reflective) transmission band give rise to serious wavelength dependent wavefront errors (WFE). The wavefront is predicted to exhibit strong WFE distortions over a very narrow wavelength range within the bandpass. This is caused by the phase-shift induced by the coating and generated by thickness in-homogeneities within the coating stack. ESA decided to remove the dielectric layers from the third flat mirror and replace these with a silver coating, and to start a detailed characterisation of the dichroic flight spare model. This model has undergone an identical manufacturing as the flight model, and the characterisation can be used for the construction of a PSF model taking account the large width of the VIS band. The new optical design would give a higher out of band transmission and a slightly lower throughput but within acceptable margins.

All flight model optical elements were delivered to ADS in Toulouse in the course of 2019 and 2020. It was found that the third mirror in the Korsch telescope did not meet the required optical performance regarding stray light due to an out of spec surface roughness. ESA decided to replace the M3 FM with the M3 STM refurbished as flight spare, which had to be polished and coated. The M3 FM was still used to carry out the alignment of the telescope before the M3 replacement was delivered in the course of 2020.

\subsection{Visual instrument (VIS)}

The PLM part of the VIS instrument [4] consists of focal plane assembly (FPA) of $364 \mathrm{k} \times 4 \mathrm{k}$ custom CCD sensors, their readout electronics and power supplies, a readout shutter unit (RSU), and a calibration unit (CU). The FPA was shipped to ADS at the end of 2019, for formal delivery in February 2020.

The RSU was delivered later, after a replacement of the gear in the unit. Even though the lifetime tests of the qualification model of the RSU indicted an increased torque within the specification margins beyond of the end of its nominal lifetime, visual inspection revealed a significant wear of the gear raising the risk of cold welding. It was decided to replace the gear in the flight model with new gear with an improved tooth design, because this could be done with no impact on the project schedule.

\subsection{Near Infrared Spectrometer and Photometer (NISP)}

The NISP instrument [5] provides a slitless spectrometer configuration with a set of four infrared grisms with one blue [920-1250 nm] and three red [1200-1850 nm] grisms, and an imaging photometer configuration with three infrared photometric bands (Y, J, H). The focal plane assembly of the instrument contains $162 \mathrm{k} \times 2 \mathrm{k}$ HgCdTe H2RG sensors with 2.3 micron cutoff wavelength, each connected through a cryo flex cable and a sensor chip electronics (SCE) unit, a SIDECAR ASIC in a custom package. Due to failure to meet thermal cycle requirements NASA decided to redesign and replace the SCE units while going ahead with the delivery of the sensor units for ground testing. The redesign, manufacturing and testing of the SCEs were performed within a short period of time, and could be delivered on time with no major impact on the overall development schedule.

The NISP instrument flight model was assembled and thermal-vacuum tested at the Laboratoire d'Astrophysique de Marseille, and shipped to ADS in Toulouse in April 2020. The instrument level tests of the complete optical mechanical assembly at thermal vacuum showed good overall performances beyond the required values in terms of throughput and infrared imaging. Tests of the three red grisms which provide the slitless dispersions at 0,180 , and 270 deg orientations in the 1200-1850 nm wavelength range indicated an anomalous performance of the 270 deg grism. This grism caused a significant point- and line-spread increase beyond margins towards the long wavelength end of the spectra. After investigation of several likely options by the EC and ESA, it was concluded that the anomaly can be mitigated without 
loss of spectroscopy performance by changing the nominal instrument observing sequence using the two 0 and 180 deg grisms operated at two dispersion angles each. The mitigation fully enables the top level science requirements, without negative impact on the survey and with no impact on the development schedule.

\section{OPERATIONS}

\subsection{Mission operations}

The operations ground segment (OGS) is based on the Euclid Mission Operations Centre (MOC) located in ESOC (Darmstadt, Germany). Their operations will start with the launch and early operations phase (LEOP) followed the commissioning phase of three months. During the nominal phase the OGS will operate a highly autonomous satellite with a 4 hours daily ground contact window for tele-commanding and reception of housekeeping data in the X-band, and reception of the science data in the K-band. The high gain antenna of the satellite is pre-commanded to repoint always to the operational ground station during the satellite pointing slews between the astronomical observation blocks. This facilitates the scheduling of the daily tele-commanding period.

Two $35 \mathrm{~m}$ deep space antennas of ESA in Malargüe and Cebreros have been equipped for the reception of the science data in the K band at $25.5-27 \mathrm{GHz}$, and have been declared operational for Euclid. Depending on the seasonal visibility of the spacecraft, one of these stations will be deployed for the daily operations.

A system validation test conducted in October 2019 validated the CFDP protocol (see section 3.2) between the spacecraft mass memory unit (MMU) and ground, and a subsequent test campaign done in July 2020 validated the file transfer from the instruments to the MMU and ground. Due to the high data volumes to be transferred and the susceptibility to losses during the RF transfer in $\mathrm{K}$ band, Euclid will be the first scientific mission of ESA applying this hand-shaking protocol as well as a real "file handling", both on-board and for transfer to ground (such as copy, move, open, and close of files). The MOC development includes spacecraft to ground interfaces and ground interfaces from MOC to the science operations. The testing of the interfaces with the science operations is commenced by the end of 2020 and progressing according to schedule.

\subsection{Science operations}

The science operations are conducted from the Euclid Science Operations Centre (SOC) in ESAC located near Madrid, Spain. The science operations will start with the performance verification phase which fill the last two months of the commissioning period. During this phase the SOC will coordinate the operations and manage the observations schedule. The Euclid Survey System (ESS) has been developed by the SOC for the scheduling of the observations and to monitor their execution [6]. The SOC will start and host the operations of the Euclid Archive System, which will store all Euclid data and related information. The SOC quick look analysis (QLA) system will be available for a timely assessment of the raw science data.

At least two weeks before the end of the performance verification phase, the reference survey definition (RSD) is delivered by the EC to the SOC for further processing in the ESS. The RSD is a list of pointings defining the Euclid nominal survey, and includes the pointings for the wide survey, deep survey as well as the dedicated routine calibration observations, and takes note of the monthly provision for platform maintenance. The SOC will verify the adequacy of the RSD and can perform re-planning of parts of the survey in case of change requests in the calibration sequence, or in case of contingencies. It controls the configuration of the RSD.

Every planning period of four weeks the SOC prepares a scheduled survey request (SSR) covering 28 days of survey and delivers this to the MOC with a lead time of 5 weeks before the execution of the first requested pointing. The SSR is composed of two distinct sub-products, one comprising the pointing requests, to be processed by Flight Dynamics at MOC, and one comprising the requests for the planned instrument commands. The duration of a complete planning cycle will last about 10 weeks, if we include one week of preparation by the SOC before submission of the survey schedule request. These timescales assumes long stable observing periods as demanded for a survey mission. In case of contingencies, a fast replanning cycle can be executed. In case no repointing is involved this can be executed in a matter of days, otherwise a lead time of two weeks must be taken into account. 


\section{SCIENCE GROUND SEGMENT}

The science ground segment (SGS) is a joint EC and ESA collaboration which consists of 10 science data centres (SDCs) including ESAC where the processing pipelines are executed and the scientific data products are generated, 9 Operational Units (OUs) which are formed by groups of scientists who are developing the pipeline processing modules called the Processing Functions (PFs), and the Instrument Operations Team (IOT) which takes care of the monitoring of the instrument health and instrument maintenance. The development schedule of the SGS closely follows that of the MOC and industry to align the overall ground segment system validation tests.

The Agile based software development is progressing along with Information Technology (IT) and Science Challenges (SCs) [7]. The IT challenges exercise the infrastructure elements to ensure that the SGS system can support an SC. The SCs exercise increasingly larger subsets of the SGS taking a simulated area of the sky as starting point. SC3 was completed at the time of the M-CDR, and the last challenge (SC8) is kicked-off in April 2020. SC8 will exercise all 10 PFs by processing of a large fraction of the simulated Euclid sky. The science challenges do not check the scientific performance of the processing functions. This will be done during a science performance verification test.

The Euclid Archive System (EAS) is meant to store and index the many petabytes of data residing in the local archives of the SDCs. Eventually, after the nominal mission it is expected that all Euclid related data are stored in the central data repository at ESAC. The EAS consists of three subsystems: the Data Processing System (DPS), the Distributed Data Storage (DDS) and the Science Archive System (SAS). In line with the development plan, a validated version of the DPS will be transferred in 2020 from its main development institute (RUG, Groningen) to the computing systems at SOC in ESAC where it will be operated. The DPS development will continue at RUG.

The Euclid mission is designed to deal with a daily downlink volume of a maximum of 850 Gbit. During nominal operations this rate is limited to less than $760 \mathrm{Gbit} /$ day for the science data, which will be transmitted in the $\mathrm{K}$ band after on-board compression. After reception at MOC, the data are sent to the SOC where they will undergo a first processing in which the data are uncompressed, reformatted, and auxiliary information is added (Level 1 products). After a quality check and indexing in the DPS, the files are made available to the 9 data centres who will calibrate and process compact areas of sky of at least 9 observation blocks with adjacent positions. The pipeline products generated by the SDCs will be registered in the DPS. Once the products in the DPS have been validated by the SGS, they will be ingested in the SAS, which has been optimised for scientific analysis purposes.

The overall configuration processing consistency of the PFs involves a centralised parameter database, the Euclid Mission Database (MDB). The MDB is directly accessed by the processing functions and contains the instrument models which are maintained by the model owners who will ingest the latest values of the model parameters. The MDB is subject to strict configuration control, as the version of the pipelines and products rely on the contents of the MDB.

\section{CALIBRATION ACTIVITIES}

We mentioned in the introduction the driving image requirements that should be met by design. In the mission requirements flow down an allocation is reserved for the margins in the knowledge that is required to construct the models of the image parameters. The WL probe demands a PSF knowledge accuracy which is an order of magnitude better than the PSF stability the telescope design can provide. It is expected that the data obtained by the space segment enable an accurate prediction of the shape of the PSF at any position in the instrument's focal plane. The GC probe requires a minimum level of purity and completeness for the H-alpha lines, which can be detected by Euclid. These knowledge requirements drive the calibration programme of Euclid. A significant amount of mission time will be dedicated to calibration, and all observations by Euclid will contribute to the calibrations needed for the cosmological analysis.

Since the Mission CDR, the calibration working group has worked on the definition of the set of calibration requirements which flow down from the top level requirements demanded by the WL and GC probes. This was a timely exercise as it goes hand in hand with the as-built performance parameters of the satellite, telescope and instruments. The derived requirements have led to the construction of the in-flight "calibration blocks" and "calibration products", which are detailed in an centralised information base, the calibration framework (CalF). The CalF blocks provide a complete specification of the calibration observations including the suitable calibration targets and observation cadences. The CalF products describe what input data are needed to extract the calibration parameters. 
The CalF is recently used to construct the first version of the operations timeline for the performance verification phase which complies with the allocated time of 2 months. During that phase, the calibration working group, the survey working group and the instrument teams are supported by the science ground segment providing the data processing to carry out the verification of the scientific performances of the telescope and instruments, validation of the observing modes and pointing sequences, as well as the initial calibration program. The CalF also enabled the scheduling of the routine calibration observations which have to be interleaved with the survey observations during the routine phase.

The processing of observations to extract calibration information is supported by the OUs as they have the expertise regarding the calibration parameters required for the processing functions. The results shall feed back to the MDB.

\section{SURVEY DEVELOPMENT}

The survey consists of the wide survey covering a required area of 15,000 deg$^{2}$ extra-galactic sky, and a deep survey of about $40 \mathrm{deg}^{2}$ as well as the additional calibration observations. The deep survey depth shall be 2 magnitudes deeper than the Wide Survey. The field of views of the two instruments are co-aligned and cover a nominal area of at least $0.53 \mathrm{deg}^{2}$. The construction of the Euclid survey is carried out by the survey working group under the leadership of the EC. This group will deliver the RSD to the SOC.
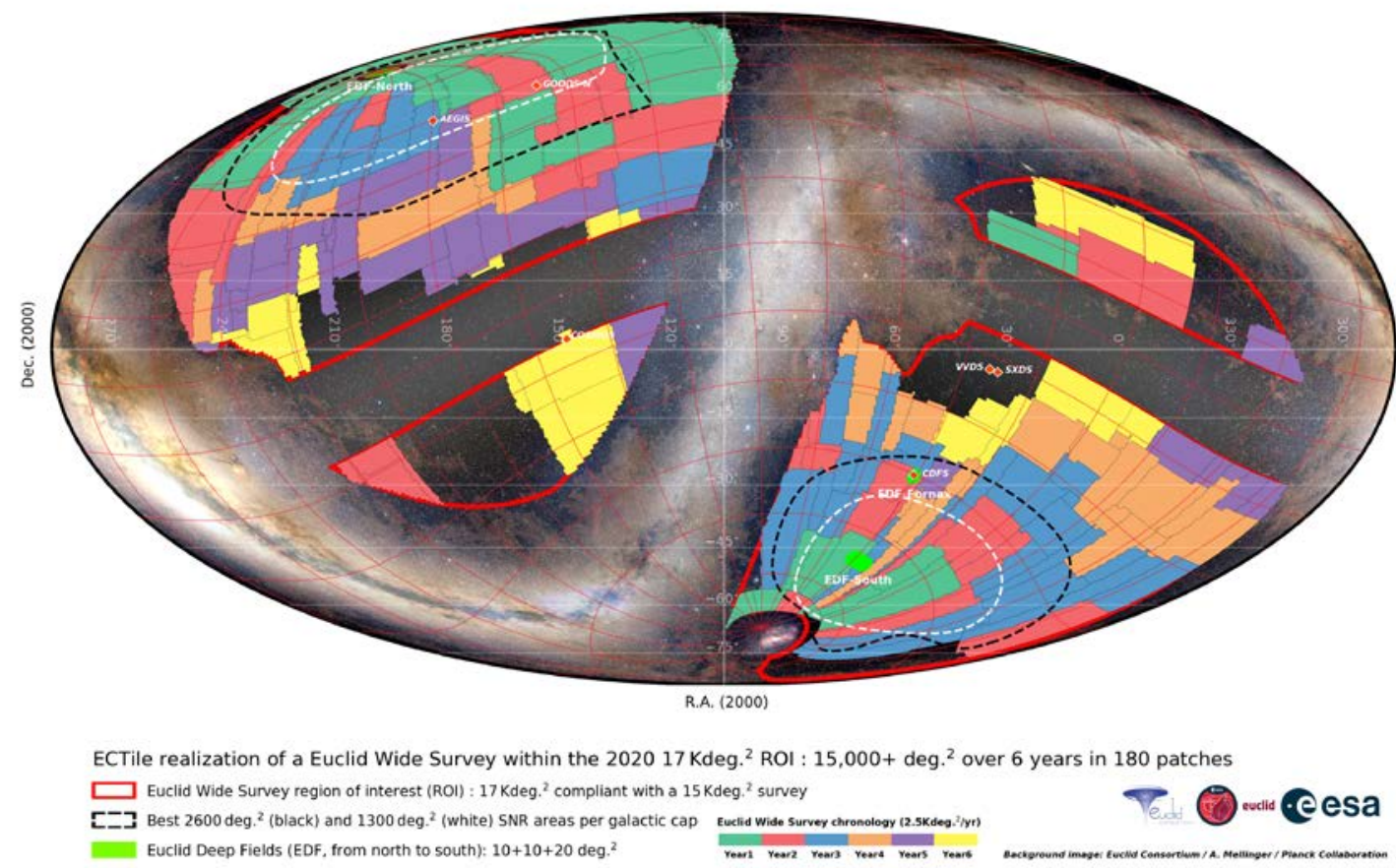

Figure 3. Equatorial map of the Euclid survey in Mollweide projection, the colours of the patches correspond to the year in which the patches are observed. The survey shown is created in 2020, with the latest mission operations requirements and routine calibration programme. The red lines delineate the region of interest (ROI), which is the area of about 17.000 deg2 of suitable extra-galactic sky for Euclid. This area excludes the galactic and ecliptic planes. The dashed areas delineate the best regions of the extra-galactic sky for Euclid and are located close to the galactic polar caps.

By design the Euclid instantaneous sky visibility area is constrained along a great circle crossing the ecliptic poles with a margin defined by the maximum azimuth rotation of the satellite with respect to the Sun. Since M-CDR a more solid definition of the slews and the slew times have become available. The construction of a standard observing block, which consists of 4 dither pointings, depends on a large number of conditions dictated by the as-built system, such as dither slew times, data transfer times, mechanisms, internal instrument calibrations, etc. In addition, to ensure that the image requirements can be achieved, thermal stability constraints on the spacecraft have been imposed on the survey by 
minimizing the amount and variation of azimuth rotation and large variations in the solar aspect angle, which is the angle between the spacecraft main axis and the normal of the ecliptic plane. These requirements enable an easier modelling of the image quality parameters, but severely limits the sequencing of the observation blocks, because the azimuth rotation is necessary to align properly the survey fields.

The deep fields programme consists three areas of the sky situated in the ecliptic north pole, in the constellation Fornax, and near the ecliptic south pole. The location and exact position of the Euclid Deep Field South were carefully considered due to the many observational constraints such as the avoidance of bright optical and infrared stars, the proximity to the Large Magellanic Cloud, interstellar extinction, scheduling feasibility, and the harmonisation with other projects/surveys. The positions of the deep fields were made public in June 2019, enabling astronomers to prepare the scientific exploitation with the Euclid observations at a pre-launch stage.

The survey working group is developing procedures and tools to be able to construct a plan which meet all constraints within the operational timescales. With the present calibration programme, it is possible to construct a wide survey which meets the area requirement of 15,000 $\mathrm{deg}^{2}$ in the 6 year nominal mission, but with few margins. In addition, the schedule cannot take into account the operational activities which depend on the conditions and system behaviour in space: molecular decontamination and telescope refocussing. ESA and the EC are presently investigating the necessary procedures in case these activities have to performed. The investigation includes and assessment of the procedures, the monitoring for such events and the impact on the wide survey and calibration program, and also relies on experiences from the Gaia mission.

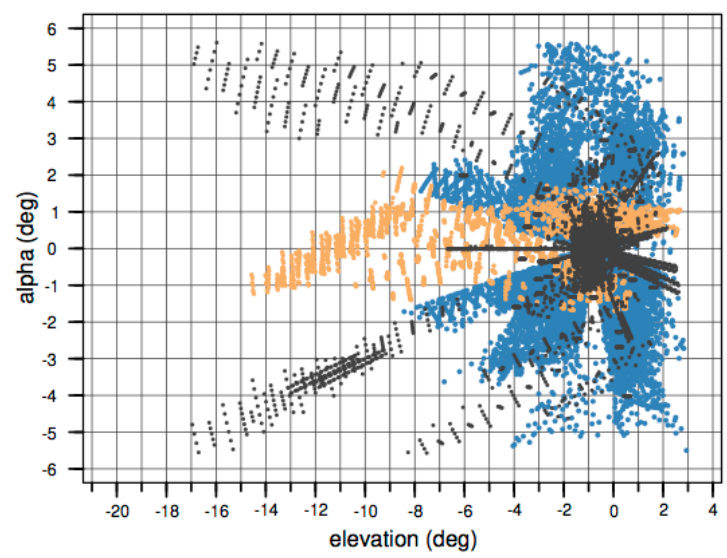

Figure 4. The distribution of the azimuth (alpha) and elevation angles for the survey presented in the previous figure. Based on the STOP analysis (see section 3.1) and in order to be able to model the PSF as accurately as required, both the absolute value as well as the variation in alpha angle from field to field were minimized for the wide survey observations, indicated by the blue dots. The orange dots represent the deep surveys, and the dedicated calibration observations are presented by the black dots.

\section{OUTLOOK}

To assess the top level science requirements of the mission, the EC has carried out science performance verification (SPV) campaigns, which are end-to-end simulations to investigate the science performances and to predict the DE-FOM of the mission. The second SPV concluded that the DE-FOM $\approx 400$ and $\Delta \gamma \approx 2 \%$ could be achieved using a combination of required values and the current best estimates for the performances at the time of M-CDR. With the as built instrument models in the MDB, the latest survey RSD, and available configuration of the SGS, the EC is preparing for the third SPV, to be kicked-off in April 2021.

The instruments qualification acceptance reviews were successfully passed for both VIS and NISP instruments in 2020. Their performances are as required. The preparations for the PLM end-to-end thermal vacuum tests are in an advanced stage to commence with the test campaign in Spring 2021. The subsequent delivery of the PLM to TAS should lead to the qualification acceptance review of the spacecraft by the end of 2021. A successful pass would open the door for the flight preparations leading to the launch in September 2022. 


\section{REFERENCES}

[1] Racca, G., Laureijs, R., Stagnaro, L., Salvignol, J.-C., Lorenzo Alvarez, J., et al. “The Euclid mission design,” Proc. SPIE 9904, 9904-O3 (2016)

[2] Boudoin, M., Mallet, F., Lavenac, J., et al. “Full-SiC EUCLID’s very large telescope,” Proc. SPIE 11180, 111801P (2019)

[3] Gaspar Venancio, L.-M., et al. "Status of the performance of the Euclid spacecraft," Proc. SPIE 11443, 11443-8 (2020)

[4] Cropper, Mark, Pottinger, S., Azzollini, R., Szafraniec, M., Awan, S., et al. "VIS: the visible imager for Euclid,” Proc SPIE 10698, 1069828-1 (2018)

[5] Maciaszek, T. "Euclid near infrared spectrometer and photometer instrument: mid phase D status," Proc SPIE 10698, 106982A (2018)

[6] Gomez-Alvarez, P., et al. “The Euclid survey planning system,” Proc. SPIE 10707, 1070712 (2018)

[7] Dubath, P. et al., "The Euclid Data Processing Challenges,” Proc. IAU Symp. 325, 73 (2017) 